\title{
Elliptic Operators in the Functional Quantisation for Gauge Field Theories
}

\author{
Sylvie Paycha \\ Département de Mathématique, Université Louis Pasteur, 7 Rue René Descartes, \\ F-67084 Strasbourg, France
}

Received: 22 December 1992/in revised form: 4 January 1994

\begin{abstract}
Given a gauge theory with gauge group $G$ acting on a path space $X$, $G$ and $X$ being both infinite dimensional manifolds modelled on spaces of sections of vector bundles on a compact riemannian manifold without boundary, it is shown that when the action of $G$ on $X$ is smooth, free and proper, the same ellipticity condition on an operator naturally given by the geometry of the problem yields both the existence of a principal fibre bundle structure induced by the canonical projection $\pi: X \rightarrow X / G$ and the existence of the Faddeev-Popov determinant arising in the functional quantisation of the gauge theory. This holds for certain gauge theories with anomalies like bosonic closed string theory in non-critical dimension and also holds for a class of gauge theories which includes Yang-Mills theory.
\end{abstract}

\section{Introduction}

Many authors have studied various aspects of the functional quantisation of gauge theories in terms of the geometric features that characterise the theory, investigating problems such as the Faddeev-Popov procedure, see e.g. [BV, FP, J, Pa], the reduction of the path space measure to a measure on the quotient space see e.g. [AGN, AHKPS, AJPS, dHP, MN, P], the problem of Gribov ambiguities see e.g. [K, S], the structure of moduli space see e.g. [FU, FT, IM, MV, NR]. The literature around these topics reveals (although not explicitly) the importance of the role of differential operators with injective symbol and of elliptic operators which naturally arise from the geometric data decribing the gauge theory. Their role is most essential for two fundamental aspects of the functional quantisation for gauge theories, namely first for the structure of the fibre bundle given by the canonical projection of the path space onto its quotient by the gauge group and the structure of the quotient space, namely the moduli space, and secondly for the Faddeev-Popov procedure. This second aspect was studied in the special case of string theory in a former paper $[\mathrm{Pa}]$. In this present article, we want to generalise these results to a larger class of gauge theories including Yang-Mills theory and bosonic string theory and show how for these gauge theories, 
the same assumption concerning the ellipticity of a differential operator given by the geometric data describing the theory, yields both the principal fibre structure for the canonical projection (and the manifold structure on the quotient) and the existence of the Faddeev-Popov determinant. In order to include the case of YangMills theory, we shall have to consider elliptic operators and differential operators with injective symbol that have coefficients in some Sobolev space, to which one can extend some essential properties - such as a Hodge-Kodaira like decomposition, Garding's inequality, spectral properties when the operator is self adjoint, that hold for elliptic operators and differential operators with injective symbol and smooth coefficients.

Let us briefly describe the contents of the present article.

Given a gauge theory with gauge group $G$ and path space $X$ which are both infinite dimensional manifolds modelled on spaces of sections of vector bundles with finite dimensional fibres on a compact Riemannian manifold without boundary, the group $G$ acting on $X$ on the right by a smooth, free and proper action

$$
\begin{aligned}
\Theta: G \times X & \rightarrow X \\
(a, x) & \rightarrow \Theta(a, x),
\end{aligned}
$$

one can consider for any $x \in X$ the differential operator-which will play a fundamental role in the following:

$$
\tau_{x}: T_{e} G \rightarrow T_{x} X
$$

tangent at $e \in G$ to the map $\theta_{x}$ that sends an element of the group into the orbit of $x$ through the action $\Theta$ :

$$
\begin{aligned}
\theta_{x}: G & \rightarrow X \\
a & \rightarrow \Theta(a, x)
\end{aligned}
$$

We shall consider right actions and set $\Theta(a, x)=x \cdot a=R_{a} x$; however the results also hold for left actions, replacing in the text right by left.

Let us remark here that if we denote by $D R_{a}: T_{e} G \rightarrow T_{a} G$ the tangent map to

$$
\begin{aligned}
R_{a}: G & \rightarrow G \\
b & \rightarrow R_{a} b=b a,
\end{aligned}
$$

then from $\theta_{x}\left(R_{a} b\right)=(x \cdot b) \cdot a$ follows that

$$
D_{a} \theta_{x}=R_{a}^{*} \tau_{x} D R_{a}^{-1}
$$

where $R_{a}^{*}$ is the pull-back of $R_{a}$ on $T X$.

Let us assume $X$ is equipped with a riemannian structure (possible weak). Under the assumption that $G$ acts on $X$ by isometries, we show (see Theorem 3.1) that if $\tau_{x}$ is an injective differential operator with smooth coefficients or with coefficients in $H^{m}$, for $m$ large enough and if the principal symbol of $\tau_{x}$ is injective (or equivalently if $\tau_{x}^{*} \tau_{x}$ is elliptic, $\tau_{x}^{*}$ denoting the adjoint of $\tau_{x}$ ), then

1) The quotient space has a smooth manifold structure (possibly infinite dimensional) and the canonical projection $\pi: X \rightarrow X / G$ yields a principal fibre bundle structure.

2) If for $x \in X, \Sigma_{x}$ is a local slice of this bundle at point $x$, and if we define for $a \in G$ the Faddeev-Popov operator $F_{a, x}^{\Sigma_{x}}$ by:

$$
\begin{aligned}
F_{a, x}^{\Sigma_{x}}: T_{e} G \times T_{x} \Sigma_{x} & \rightarrow T_{R_{a} x} X \\
(u, h) & \rightarrow D_{a} \theta_{x} \circ D R_{a} u+R_{a}^{*} h
\end{aligned}
$$


(where $R_{a}^{*} h$ is the pull-back of $h$ by $R_{a}$ ), then the Faddeev-Popov operator has a well defined determinant, for an arbitrary choice of the local slice $\Sigma_{x}$ whenever the quotient $X / G$ is finite dimensional and for a local slice $\Sigma_{x}$ orthogonal to the orbit at point $x$ whenever the quotient is infinite dimensional. This determinant is independent of the point $R_{a} x$ chosen in the orbit of $x$ and only depends on $x$ and $\Sigma_{x}$.

Let us point out here that the equivalence between the assumption on the ellipticity of the operator $\tau_{x}^{*} \tau_{x}$ and that of the injectivity of the principal symbol of $\tau_{x}$ is not true in general, but follows here from the fact that the fibres of the vector bundle on which $\tau_{x}$ and $\tau_{x}^{*}$ are defined, are finite dimensional.

These statements are a step towards a more systematic understanding of aspects of the functional quantisation procedure of gauge theories from the features of the geometric data describing the corresponding classical theory. The proof of the first of these statements is based on classical slice theorems, which we recall in the course of the paper. The second statement is a generalisation of results of [P].

The framework described above applies directly to Yang-Mills theory for which, as we shall see, the path space $X$ can indeed be equipped with a gauge (or $G$ ) invariant riemannian structure. However, in order to include a class of gauge theories with anomalies, like that of bosonic string theory in non-critical dimension, we study the general case when $X$ is equipped with a riemannian structure which is not $G$ invariant, but only $H$-invariant, $H$ being a subgroup of $G$. In our framework, $G$ is the whole symmetry group for the classical action and $H$ the symmetry group left after quantisation, i.e. the invariance group of the Riemannian structure on $X$. When $H \neq G$, a certain type of anomaly can arise as in the example of the string model in non-critical dimensions where conformal anomaly arises from the non-invariance of the riemannian structure under the action of the Weyl group. The obstruction to choosing a riemannian structure invariant under the whole symmetry group of the classical action of the theory is of course only one aspect of the problem of anomalies. In order to give a full treatment of anomalies in string theory, one also has to consider the global anomaly which arises as a topological obstruction characterised by a non-vanishing first Chern class of a line bundle built up from determinant bundles and the local or geometric anomaly which arises as an obstruction to flatness of this line bundle $[\mathrm{F}]$. However, in the framework of functional quantisation of string theory, these obstructions arise after the (Faddeev-Popov) reduction procedure from the original path integral on the infinite dimensional path space down to a finite dimensional integral over Teichmuiller parameters. Here we are interested in the first manifestation of anomalies mentioned above, namely that of the non-invariance of the riemannian structure on the infinite dimensional path space under the whole symmetry group of the classical theory before the reduction procedure takes place.

We therefore extend the framework described above to the case when $G$ is a semi-direct product of two groups $G=H \odot K$, each of them being an infinite dimensional manifold modelled on a space of sections of some vector bundle with finite dimensional fibres on a boundaryless smooth finite dimensional compact manifold. We shall assume that $G$ acts on $X$ by:

$$
\begin{aligned}
\Theta:(H \odot K) \times X & \rightarrow X \\
\left(\left(a^{\prime}, a^{\prime \prime}\right), x\right) & \rightarrow R_{a^{\prime}}\left(R_{a^{\prime \prime}} x\right)=\left(x \cdot a^{\prime \prime}\right) \cdot a^{\prime} .
\end{aligned}
$$

We shall identify the tangent space to $G$ to the product of the tangent spaces to $H$ and $K$, setting for $a=\left(a^{\prime}, a^{\prime \prime}\right)$,

$$
T_{\left(a^{\prime}, a^{\prime \prime}\right)} G=T_{a^{\prime}} H \times T_{a^{\prime \prime}} K,
$$


so that the tangent map $D_{\left(a^{\prime}, a^{\prime \prime}\right)} \theta_{x}$ splits into a sum of maps:

$$
D_{\left(a^{\prime}, a^{\prime \prime}\right)} \theta_{x}=R_{a^{\prime}}^{*} \tau_{R_{a^{\prime \prime}} x}^{\prime} D R_{a^{\prime}}^{-1}+R_{a}^{*} \tau_{x}^{\prime \prime} D R_{a^{\prime \prime}}^{-1},
$$

where $\tau_{x}^{\prime}=D_{e} \theta_{x}^{\prime}\left(\right.$ resp. $\left.\tau_{x}^{\prime \prime}=D_{e} \theta_{x}^{\prime \prime}\right), \theta_{x}^{\prime}\left(\right.$ resp. $\left.\theta_{x}^{\prime \prime}\right)$ being the restriction of $\theta_{x}$ to $H$ (resp. $K$ ).

Notice that with these conventions, $\tau_{x}=\tau_{x}^{\prime}+\tau_{x}^{\prime \prime}$, but relation (0.4) does not always hold any more. However if

$$
\tau_{R_{a^{\prime \prime}} x}^{\prime \prime}=R_{a^{\prime \prime}}^{*} \tau_{x}^{\prime \prime}
$$

then we have

$$
D_{\left(a^{\prime}, a^{\prime \prime}\right)} \theta_{x}=R_{a^{\prime}}^{*} \tau_{R_{a^{\prime \prime} x}} D R_{a}^{-1}
$$

and conversely. Here $D R_{a}$ has been identified to $D R_{a^{\prime}} \times D R_{a^{\prime \prime}}$.

Of course, if $H=G$, relation (0.8) is trivially satisfied since $K=\{e\}$. Since

$$
\tau_{R_{a^{\prime \prime} x}}^{\prime \prime}=R_{a^{\prime \prime}}^{*} \tau_{x}^{\prime \prime} \operatorname{ad}_{a^{\prime \prime}}
$$

where $\operatorname{ad}_{a^{\prime \prime}}$ is the tangent map acting on the tangent space $T_{e} K$ to the map which sends $b^{\prime \prime} \in K$ to $a^{\prime \prime} b^{\prime \prime} a^{\prime \prime-1}$ in $K$, condition (0.8) is also trivially satisfied when $K$ is commutative. Conversely, if the action of $K$ on $X$ is free and if $K$ is a (possibly infinite dimensional) connected Lie group equipped with a surjective exponential map, then from (0.8) follows the commutativity of $K$.

In the following, we shall assume that condition (0.8) is satisfied, thus making an assumption on the group $K$ which is in particular fulfilled when $K$ is abelian as in the case of bosonic string theory.

In this more general framework, we also show statements 1) and 2) from the assumption that the principal symbol of $\tau_{x}$ be injective, up to the fact that the assumption on the $G$-invariance of the riemannian structure is replaced by the $H$ invariance of this same riemannian structure on $X$.

The paper is organised as follows:

In Sect. I, we first give a slice theorem (Lemma 1.1) in the case when the manifolds involved are Hilbert manifolds, letting $G$ be semi-Hilbert Lie group, a notion which we introduced below and which generalises that of a Hilbert Lie group, including groups such as the group of diffeomorphisms on a compact manifold without boundary which are of Sobolev class. This slice theorem generalises the classical slice theorem where $G$ is a Hilbert Lie group (see e.g. [FU, FT, H, IM, NR]) and slice theorems involving the group of diffeomorphisms of Sobolev class which implicitly underlie works like [E] and [FM]. We then apply this slice theorem to the case when both manifolds $X$ and $G$ are modelled on spaces of sections of Sobolev class of some vector bundle with finite dimensional fibres on a compact boundaryless manifold, showing (in Theorem 1.2) that if $G$ acts on $X$ through a smooth, free and proper action, and if $\tau_{x}$ is an injective differential operator with injective symbol (and possibly coefficients of Sobolev class), then there is a Hilbert manifold structure on the quotient $X / G$ and a principal fibre bundle structure given by the canonical projection $\pi: X \rightarrow X / G$. This holds for a group $G$ of the form $G=H \odot K, H \neq G, H$ acting on $X$ through isometries, $X$ being equipped with a weak $L^{2}$ riemannian structure.

In Sect. II, we deduce from the above slice theorem a slice theorem (Lemma 2.1) for the case of manifolds which are inductive limits of Hilbert manifolds, namely I.L.H. manifolds, letting $G$ be an inverse limit of a family of semi-Hilbert Lie groups. Applying it to the case when both $X$ and $G$ are modelled on spaces of smooth 
sections of some vector bundle on a compact boundaryless surface, we show (up to some additional technical assumptions explicitly given in Theorem 2.2 and discussed in a remark following the theorem) that if $G$ acts on $X$ through a smooth, free and proper action and if the operator $\tau_{x}$ is an injective differential operator with injective symbol (and smooth coefficients), then the quotient space is an I.L.H. manifold and the canonical projection yields an I.L.H. principal fibre bundle. Here again, this holds in the general case when $G=H \odot K, H$ acting on $X$ through isometries, $X$ being equipped with a weak $L^{2}$ riemannian structure.

In Sect. III, we prove (Theorem 3.1) that under the same above assumptions (namely that the action of $G$ on $X$ is smooth, free and proper and that $\tau_{x}$ is an injective differential operator with injective symbol, $G=H \odot K, H$ acting on $X$ through isometries), and for a given local cross-section at point $x$ of the principal bundle $X \rightarrow X / G$, one can define the Faddeev-Popov determinant which is independent of the point in the orbit of $x$ for the action of $H$. We show that if the quotient $X / G$ is finite dimensional, then the determinant of this operator is well defined for any choice of the local section, and if the quotient is infinite dimensional, the determinant is well defined for any local section orthogonal to the orbit of $x$ for the action of $G$.

For the sake of clarity, we chose to leave certain technical devices and one proof out of the main text and to refer the reader to the Appendices. In particular, we shall need a generalisation to a class of positive self adjoint elliptic operators with coefficients of Sobolev class of the notion of regularised determinant, which we give in Appendix A.2 using results of $[\mathrm{C}, \mathrm{KR}]$ and the presentation of regularised determinants in [AJPS, Pa] done for elliptic operators with smooth coefficients.

All along the paper, we illustrate the theorems by Yang-Mills theory and the theory of closed bosonic strings.

\section{Differential Operators with Injective Symbol and Slice Theorems in the Hilbert Case}

We shall call a right (resp. left) semi-Hilbert Lie group a Hilbert manifold with a topological group structure such that the right (resp. left) multiplication is smooth. A typical example of a right semi-Hilbert Lie group is, for $s$ large enough, the group of diffeomorphisms $\mathscr{V}^{s}(M)$ of a smooth compact riemannian manifold $M$ without boundary described in $[\mathrm{O}, \mathrm{E}]$ for example. If $n$ is the dimension of the manifold $M$ and if $H^{s}(M \times M)$ denotes the space of sections of the bundle $M \times M$ over $M$ in the Sobolev class $H^{s}$, then by classical Sobolev embedding theorems, for $s>1+\frac{n}{2}$, we have the inclusion $H^{s}(M \times M) \subset C^{1}(M \times M)$, where $C^{1}(M \times M)$ denotes the space of sections of $M \times M$ which are $C^{1}$ and the inclusion is continuous.

Let us set

$\mathscr{D}_{C^{1}}(M) \equiv\left\{f \in C^{1}(M \times M)\right.$, such that $f$ has an inverse and $\left.f^{-1} \in C^{1}(M \times M)\right\}$ and

$$
\mathscr{D}^{s}(M) \equiv H^{s}(M \times M) \cap \mathscr{D}_{C^{1}}(M) .
$$

Since $\mathscr{D}_{C^{1}}(M)$ is open in $C^{1}(M \times M), \mathscr{D}^{s}(M)$ is also open in $H^{s}(M \times M)$, and it is a Hilbert manifold modelled on $H^{s}(M \times M)$. The r.h.s. multiplication in $\mathscr{D}^{s}(M)$ defined for $h \in \mathscr{D}^{s}(M)$ by:

$$
\begin{aligned}
R_{h}: \mathscr{D}^{s}(M) & \rightarrow \mathscr{D}^{s}(M) \\
f & \rightarrow f \circ h
\end{aligned}
$$


is $C^{\infty}$, but the 1.h.s. multiplication defined for $h \in \mathscr{D}^{s+l}(M)$ by

$$
\begin{aligned}
L_{h}: \mathscr{D}^{s}(M) & \rightarrow \mathscr{D}^{s}(M) \\
f & \rightarrow h \circ f
\end{aligned}
$$

is only of class $C^{l}$ so that $\mathscr{D}^{s}(M)$ defines is a semi-Hilbert Lie group in the above sense.

The basic theorem underlying the subsequent results is the following generalisation to the case of a semi-Hilbert Lie group acting on a Hilbert manifold of the classical slice theorem concerning the action of a Hilbert Lie group on a Hilbert manifold (which one can find for example in [B, Bo, FM, FT, IM]). It can be proved along the lines of a classical proof used in the finite dimensional case (see e.g. [Br, V]).

Lemma 1.1. Let $G$ be a right (resp. left) semi-Hilbert Lie group acting on $X$ by $a$ right (resp. left) action on a $C^{\infty}$ Hilbert manifold through a right (resp. left) action $\Theta$. Assume that
(Ao) $\left\{\begin{array}{l}- \text { The action is smooth, free and proper, } \\ - \text { For any } x \in X, \text { the map } \theta_{x} \text { is an immersion, } \\ \text { (i.e. the tangent map } D \theta_{x} \text { is injective and its image is closed in } T X \text { ) }\end{array}\right.$

Then

1) $X / G$ is a Hilbert manifold.

2) The canonical projection $\pi: X \rightarrow X / G$ yields a $C^{\infty}$ principal fibre bundle structure with structure group $G$.

Proof. Since the classical proof for a Hilbert Lie group acting on a Hilbert manifold extends to the case of a semi-Hilbert Lie group acting on a Hilbert manifold without any major modification, we leave out the proof for the moment, which is explicitly done in Appendix A.1.

When the manifolds $X$ and $G$ are modelled on spaces of sections of vector bundles with fibres of finite dimension on a compact boudnaryless riemannian surface, the assumption that $\theta_{x}$ be an immersion appears as a natural consequence of some condition on the principal symbol of the operator $D_{a} \theta_{x}$, namely that it should be injective.

All along the paper, $M$ denotes a finite dimensional compact boundaryless $C^{\infty}$ riemannian manifold. We shall consider $C^{\infty}$ vector bundles on $M$ that have finite dimensional fibres, chosen in such a way that they naturally inherit a euclidean structure from the Riemannian structure on $M$. Typically, we shall choose bundles such as the trivial bundle $M \times \mathbb{R}$, tensor bundles $T M^{k} \otimes T^{*} M^{l}$, or bundles built up from an additional principal fibre bundle $\mathscr{P}$ on $M$ with structure group $\mathscr{G}$ like $\mathscr{P} \times \mathscr{G}, \mathscr{g}, \mathscr{P} \times \mathscr{g} \otimes T^{*} M$, where $\mathscr{G}$ is a finite dimensional compact semi-simple matrix Lie group and $\mathbf{g}$ the Lie algebra of $\mathscr{G}$.

Let $\mathscr{T}$ be a such vector fibre bundle on $M$ and let us denote by $H^{s}(\mathscr{T})$ the space of sections of class $H^{s}$ of $\mathscr{V}$, i.e. the closure of the space of $C^{\infty}$ sections for an $H^{s}$ scalar product induced by the metric on $M$.

Theorem 1.2. Let $\mathscr{E}, \mathscr{H}, \mathscr{K}$ be $C^{\infty}$ vector fibre bundles on $M$ (of dimension $n$ ) with finite dimensional fibres. Let $X$ be a Hilbert manifold modelled on $H^{s}(\mathscr{E})$ for some $s \in \mathbb{N}$. Let $k, h$ be two integers such that $\frac{n}{2}+r+1 \geq s \geq r$, where $r=\max (h, k)$. 
Let $G$ be a right (resp. left) semi-Hilbert Lie group which is a semi-direct product of two groups, $G=H \odot K, H$ and $K$ being Hilbert manifolds modelled on $H^{s+h}(\mathscr{H})$ and $H^{s+k}(\mathscr{K})$ respectively. $\mathscr{G}$ acts on $X$ through an action $\Theta$ :

$$
\begin{aligned}
\Theta:(H \odot K) \times X & \rightarrow X \\
\left(\left(a^{\prime}, a^{\prime \prime}\right), x\right) & \rightarrow R_{a^{\prime}}\left(R_{a^{\prime \prime}} x\right) .
\end{aligned}
$$

We shall assume as before, that for $a=\left(a^{\prime}, a^{\prime \prime}\right) \in G$ :

$$
D_{a} \theta_{x}=R_{a^{\prime}}^{*} \tau_{a^{\prime \prime} x} D R_{a}^{-1} .
$$

Moreover, we assume $X$ is equipped with a weak $L^{2}$ riemannian structure invariant under the action of $H$.

Under the following assumption

$$
\text { (A) }\left\{\begin{array}{l}
\text { - The action } \Theta \text { is smooth, free and proper } \\
- \text { For each } x \in X \text { the map } \tau_{x}: T_{e} G \rightarrow T_{x} X \\
\text { is an injective differential operator of order } r \text { with injective symbol } \\
\text { and coefficients of Sobolev class } m \text { with } m>\frac{n}{2}+2 r .
\end{array}\right.
$$

Then

1) $X / G$ is a Hilbert manifold.

2) The canonical projection $\pi: X \rightarrow X / G$ yields a $C^{\infty}$ principal fibre bundle structure with structure group $G$.

Remark. If $G=H$, relation (1.3) is of course trivially satisfied.

Proof. In order to apply Lemma 1.1, we only need to check that $\theta_{x}$ is an immersion. For the sake of clarity, we first prove that $\theta_{x}$ is an immersion in the simpler case $H=G$. The fact that $D \theta_{x}$ is injective follows from the relation $D_{a} \theta_{x}=R_{a}^{*} \tau_{x} D R_{a}^{-1}$ since $\tau_{x}$ is itself injective.

Let us now check that $\operatorname{Im} D_{a} \theta_{x}$ is closed. For this we first check that $\operatorname{Im} \tau_{x}$ is closed. Since $\tau_{x}$ has injective symbol and since $m>\frac{n}{2}+2 r$, by Theorem A.2.2, Appendix A.2, the operator $\tau_{x}$ has a closed image w.r.t. the $L^{2}$ topology on $T X$ induced by the weak riemannian structure on $X$ and $H^{s}(\mathscr{E})=\operatorname{Im} \tau_{x} \oplus \operatorname{Ker} \tau_{x}^{*}$. From this, we deduce that $\operatorname{Im} D_{a} \theta_{x}$ is closed in $T_{R_{a} x} X$ w.r.t. the $L^{2}$ topology and that there is an orthogonal splitting $T_{R_{a}} X=\operatorname{Im} D_{a} \theta_{x} \oplus \operatorname{Ker}\left(D_{a} \theta_{x}\right)^{*}$ since $D_{a} \theta_{x}=R_{a}^{*} \tau_{x} D R_{a}^{-1}$ and since the metric is $G$ invariant. From this follows that this image is also closed w.r.t. the Hilbert manifold topology (see e.g. [KV, Lemma 3.1.6]) on $X$.

The proof in the general case $G \neq H$ goes in the same way using relation (1.3) and the fact that for $a \in G, \operatorname{Im} \tau_{R_{a} x}$ is closed.

Examples. 1. We first illustrate this theorem in the case $G=H$ by Yang-Mills theory.

Set $n=\operatorname{dim} M$. Let $\mathscr{P}=\mathscr{P}(M, \mathscr{G})$ be a smooth principal fibre bundle on $M$ with structure group $\mathscr{G}$, a compact semi-simple matrix Lie group. Let $E_{\text {ad }}=\mathscr{P} \times \mathscr{G}$ be the bundle with the Lie algebra $\mathbf{g}$ of $\mathscr{G}$ as a standard fibre and with the adjoint action of $\mathscr{G}$ on $\mathbf{g}$. We set $E_{\mathscr{G}}=\mathscr{P} \times_{\mathscr{G}} \mathscr{G}$. Take $\mathscr{E}=E_{\text {ad }} \odot T M^{*}$, the bundle of 1 -forms on $M$ with values in $E_{\text {ad }}$ and $\mathscr{F}=E_{\text {ad }}$.

We first describe the Hilbert manifold $X$. Set for $s>\frac{n}{2}+1, X$ to be the space $\mathscr{A}$, of connections on $\mathscr{P}(M, \mathscr{G})$ of Sobolev class $H^{s}$ (see for example [KR, FU] for an explicit description of $\mathscr{b}_{s}$ ). $\mathscr{b}_{s}$ is a closed affine spaces in the space 
$H^{s}\left(E_{\mathrm{ad}} \odot T^{*} M\right)$ of $H^{s}$ Sobolev sections of the bundle $\mathscr{E}$, i.e. the space of $\mathrm{g}$-valued $H^{s}$ 1-forms on $\mathscr{P}$ (see e.g. [FU, KR, MV]) and by the Sobolev embedding theorems, $\mathscr{A}_{s}$ is contained in the space of $C^{1}$ connections on $\mathscr{P}(M, \mathscr{G})$. The subspace $\overline{\mathscr{A}}_{s}$ of irreducible connections in $t^{s}$ (see e.g. [FU], Chap. 3 for a definition of irreducible connection) is a Hilbert manifold modelled on $H^{s}\left(E_{\mathrm{ad}} \otimes T^{*} M\right)$. We shall set $X=\overline{\mathscr{A}}_{\mathrm{s}}$.

Let us now describe the group $G$. Let $H^{s}\left(E_{G}\right)$ be the group formed by the sections of Sobolev class of the bundle $E_{\mathscr{G}}$ or equivalently the group of fibre preserving maps $s: \mathscr{P} \rightarrow \mathscr{P}$ which satisfy $\pi \circ s=\pi$ and $s(p . g)=s(p) g$ for $p \in \mathscr{P}, g \in \mathscr{G}$ of class $H^{k}$ (see e.g. [FU, KR]) with pointwise multiplication. For $s>\frac{\operatorname{dim}_{\mathscr{P}}}{2}+1$, $H^{s}\left(E_{\zeta}\right)$ is a Hilbert Lie group modelled on $H^{s}\left(E_{\mathrm{ad}}\right)$ (the space of $H^{s} g$-valued equivariant functions on $\mathscr{P}$ ) and a closed topological subgroup of $\mathscr{D}^{s}(\mathscr{P})$ (see e.g. $[\mathrm{KR}]$ ), the group of all $H^{s}$ diffeomorphisms which itself is not a Hilbert Lie group but only a semi-Hilbert Lie group. Let $\mathscr{Z}_{S}$ be the centre of $H^{s}\left(E_{\mathscr{G}}\right)$. The group $G_{s}=H^{s}\left(E_{G}\right) / \mathbb{Z}_{s}$ is also a Hilbert Lie group modelled on $H^{s}\left(E_{\mathrm{ad}}\right)$ (see Theorem 2.18, [MV]). We shall set $G=G_{s+1}$.

The right action of $H^{s+1}\left(E_{\mathrm{ad}}\right)$ on . $\mathrm{t}_{s}$ induces a right action of $G$ on $X$ :

$$
\begin{aligned}
\Theta: G_{s+1} \times \mathscr{t}_{s} & \rightarrow \mathscr{A}_{s} \\
(g, A) & \rightarrow R_{A} g=A+g^{-1} d_{A} g
\end{aligned}
$$

where $d_{A}: H^{s+1}\left(E_{\mathrm{ad}}\right) \rightarrow H^{s}\left(E_{\mathrm{ad}} \otimes T M^{*}\right)$ is the exterior covariant derivative induced by the connection $A$.

There is a weak $L^{2} G$-invariant metric on $\mathscr{A}^{s}$ (see e.g. [KR, par. 2.3]) given by

$$
\langle\alpha, \beta\rangle=\int_{\mathscr{P}}(\alpha(p), \beta(p)) d \mu(p)
$$

where $\mu$ is a smooth $\mathscr{G}$-invariant measure on $\mathscr{P}, \alpha, \beta$ are $g$ valued one forms on $\mathscr{P}$ and $(\cdot, \cdot)$ the bundle metric in $g \otimes T^{*} \mathscr{P}$.

The action of $G=G_{s+1}$ on $X=\overline{\mathscr{C}_{s}}$ is $C^{\infty}$ (see e.g. [MV, p. 465]), free (see e.g. $\left[\mathrm{MV}\right.$, p. 466]) and proper (see e.g. [KR, Theorem 2.4.9]). For $A \in \mathscr{f}_{s}$, the map $\tau_{A}$ tangent at $1 \in G$ to:

$$
\begin{aligned}
\theta_{A}: G & \rightarrow X \\
g & \rightarrow R_{g} A
\end{aligned}
$$

coincides with $d_{A}$.

For $A \in \mathscr{b}_{s}$, the operator $d_{A}$ is a differential operator of order 1 with coefficients of Sobolev class $s-1$. With the notations of Theorem 1.2, we set $m=s-1$, $r=h=1$.

For $s>\frac{n}{2}+3$ and $A \in \overline{\mathscr{A}}_{s}$, the operator $d_{A}$ lies in $I n j^{s-1}\left(E_{\mathrm{ad}}, E_{\mathrm{ad}} \otimes T^{*} M\right)$ (see Appendix A.2 for a definition), since $d_{A}^{*} d_{A}=L+D$ where $L$ is an elliptic operator with smooth coefficients and $D$ a differential operator of lower order with coefficients in $H^{s-2}$ (see e.g. [KR, par. 3.1]). The injectivity of the map $\tau_{A}$ follows from the existence of an exponential map and the freedom of the action as in the proof of Lemma 1.1.

The assumptions of Theorem 1.2 are therefore satisfied and we conclude that for $s>\frac{n}{2}+3 . \overline{\mathscr{A}}_{s} / G_{s+1}$ is a Hilbert manifold and that $\overline{\mathscr{A}}_{s} \rightarrow \overline{\mathscr{A}}_{s} / G_{s+1}$ is a principal fibre bundle with structure group $G_{s+1}$. 
2. We now illustrate Theorem 1.2 in the case $G \neq H$ by the theory of closed bosonic strings.

Take $n=\operatorname{dim} M=2$ and $M$ of genus $p>1$. Let us set $\mathscr{E}=S^{2} T^{*}=$ $T^{*} M \otimes_{s} T^{*} M$, the symmetric tensor product of the contangent bundle on $M$ with itself. Let for $s>1, X$ be the manifold

$$
\mathscr{C l}^{s}(M) \equiv\left\{g \in H^{s}\left(S^{2} T^{*}\right), \operatorname{det} g>0\right\}
$$

of $H^{s}$ riemannian metrics on $M$, which is a Hilbert manifold modelled on $H^{s}(\mathscr{E})$.

We now define the group $G$. Let $\mathscr{D}_{0}^{s+1}(M)$ be the semi-Hilbert Lie group defined as the connected component of the identity in $\mathscr{D}^{s+1}(M)$ defined in (1.1); it is a Hilbert manifold modelled on $H^{s+1}(T M)$ [FT]. Let $\mathscr{W}(M)=\left\{e^{\phi}, \phi \in H^{s}(M, \mathbb{R})\right\}$ and let the group $G$ be semi-direct product of these two groups,

$$
G=\mathscr{D}_{0}^{s+1}(M) \odot \mathscr{H}^{\cdot s}(M) .
$$

$G$ is of the form $H \odot K$, where $H=\mathscr{Q}_{0}^{s+1}(M), K=\mathscr{W}^{s}(M)$ are modelled on $H^{s+1}(\mathscr{H})$ and $H^{s}(\mathscr{K})$, respectively, taking $\mathscr{H}=T M$ and $\mathscr{K}^{\circ}=M \times \mathbb{R}$.

The group $G$ acts on $X=\mathscr{A l}^{s}(M)$ by a right action:

$$
\begin{aligned}
\Theta:\left(\mathscr{D}_{0}^{s+1}(M) \odot \mathscr{W}^{s}(M)\right) \times \mathscr{H}^{s}(M) & \rightarrow \mathscr{M}^{s}(M) \\
\left(\left(f, e^{\phi}\right), g\right) & \rightarrow f^{*} e^{\phi} g .
\end{aligned}
$$

The action $\Theta$ is $C^{\infty}$, free and proper (see e.g. [AJPS]). For $g \in \mathscr{M l}^{s}(M)$, the operator $\tau_{g}$ tangent at point $(\mathrm{Id}, 1) \in G$ to the map:

$$
\begin{aligned}
\theta_{g}: \mathscr{D}^{s+1}(M) \odot \mathscr{W}^{s}(M) & \rightarrow \mathscr{M}^{s}(M) \\
\left(f . e^{\phi}\right) & \rightarrow f^{*} e^{\phi} g
\end{aligned}
$$

is of the form

$$
\begin{aligned}
\tau_{g}: H^{s+1}(T M) \times H^{s}(M, \mathbb{R}) & \rightarrow H^{s}\left(S^{2} T^{*}\right) \\
(u, \lambda) & \rightarrow \nabla_{g} u+\lambda g,
\end{aligned}
$$

where $\left(\nabla_{g} u\right)_{a b}=\nabla_{a} u_{b}+\nabla_{b} u_{a}$. Furthermore, with the notations of the introduction, we check that for $\lambda \in H^{s}(\Lambda, \mathbb{R}), \tau_{e^{\phi}{ }_{g}}^{\prime \prime} \lambda=e^{\phi} \tau_{g}^{\prime \prime} \lambda$, since $\tau_{g}^{\prime \prime} \lambda=\lambda g$ so that $D_{\left(f, e^{\phi}\right)} \theta_{g}\left(f^{*-1} u . e^{-\phi} \lambda\right)=f^{*} \tau_{e^{\phi} g}(u . \lambda)$ and relation (1.3) is satisfied. As we pointed out in the introduction, this follows from the commutativity of $K=\mathscr{W}^{s}(M)$.

There is a weak $L^{2}$ Riemannian metric on. $\mathscr{L}^{s}(M)$ induced at point $g \in \mathscr{L}^{s}(M)$ by the scalar product

$$
\langle h, k\rangle_{L^{2}}=\int_{M} \sqrt{g} h \cdot k
$$

(where, for two tensor fields $t$ and $t^{\prime}$ the product $t \cdot t^{\prime}$ denotes the contracted product using the metric $g$ ). It is clearly $H=\mathscr{D}_{0}^{s+1}(M)$ invariant. For any metric $g$, there is an orthogonal splitting w.r.t. the $L^{2}$ scalar product induced on $T_{g} \cdot \mathscr{C l}^{s}(M) \simeq H^{s}\left(S^{2} T^{*}\right)$ by this riemannian structure:

$$
H^{s}\left(S^{2} T^{*}\right)=H^{s}(M, \mathbb{R}) g \oplus H^{s}\left(S_{0}^{2} T^{*}\right)
$$

through which one can identify $T_{g} \mathscr{C l}^{s}(M)$ to $H^{s}(M, \mathbb{R}) \times H^{s}\left(S_{0}^{2} T^{*}\right)$, where $H^{s}\left(S_{0}^{2} T^{*}\right)$ is the space of $H^{s}$ traceless two symmetric tensors on $M$ and where for a symmetric two tensor $t$, the trace w.r.t. the metric $g$ is given by $\operatorname{tr}_{g} t=g^{a b} t_{a b}$. 
Define for $g \in \mathscr{M}^{s}(M)$ the operator

$$
\begin{aligned}
P_{g}: C^{\infty}(T M) & \rightarrow H^{s-1}\left(S_{0}^{2} T^{*}\right) \\
u & \rightarrow \nabla_{g} u-\frac{1}{2} \operatorname{tr}_{g}\left(\nabla_{g} u\right) g .
\end{aligned}
$$

It is a differential operator with injective symbol as can be seen going over to local coordinates (see e.g. [AJPS], Chap. 4). It is easy to check that for any integer $s>3$, $P_{g} \in I n j^{s-1}\left(T M, S_{0}^{2} T^{*}\right)$ since $P_{g}$ is of order 1 .

The operator $\tau_{g}$, interpreted as a matrix operator from $H^{s}(T M) \times H^{s}(M, \mathbb{R})$ to $H^{s}\left(S_{0}^{2} T^{*}\right) \times H^{3}(M, \mathbb{R})$ reads

$$
\tau_{g}=\left[\begin{array}{cc}
P_{g} & 0 \\
\frac{1}{2} \operatorname{tr}_{g}(\cdot) g & g
\end{array}\right]
$$

since $\tau_{g}(u, \lambda)=P_{g} u+\frac{1}{2} \operatorname{tr}_{g}\left(\nabla_{g} u\right)+\lambda g$. One can show that the operator $P_{g}$ is injective checking on one hand that its kernel is given by holomorphic vector fields and on the other hand, using the Riemann Roch theorem, that there are no non-zero holomorphic vector fields on a Riemann surface of genus $p>1$. The fact that it has injective symbol follows from the injective of the symbol of $P_{g}$. Setting $m=s-1, k=1$, so that for any integer $s>3$ (recall that $n=2$ ), $\tau_{g} \in \operatorname{Inj}^{s-1}(\mathscr{F}, \mathscr{E}$ ).

The assumptions of Theorem 1.2 are hence all satisfied and for $s>3$,

$$
\mathscr{C l}^{s}(M) / \mathscr{D}_{0}^{s+1}(M) \text {. }
$$

$\mathscr{W}^{s}(M)$ is therefore a $C^{\infty}$ Hilbert manifold and the projection

$$
\mathscr{H}^{s}(M) \rightarrow \mathscr{C l}^{s}(M) / \mathscr{D}_{0}^{s+1}(M) \odot \mathscr{W}^{s}(M),
$$

a principal fibre bundle with structure group $\mathscr{D}_{0}^{s+1}(M) \odot \mathscr{W}^{s}(M)$.

\section{Differential Operators with Injective Symbol and the Slice Theorem in the I.L.H. Case}

In this section, we want to extend the results of Sect. I to the case when $X$ and $G$ are I.L.H. manifolds. We refer the reader to Appendix A.3 for the relevant definitions concerning I.L.H. structures, in particular I.L.H. manifolds, I.L.H. Lie groups and I.L.H. fibre bundles. We define here I.L.H. actions.

Definition. Let $X$ be a $C^{\infty}$ I.L.H. manifold, $G=\bigcap_{\imath \in \mathbb{N}} G_{\imath}$ and I.L.H. Lie group acting on $X=\bigcap_{i \in \mathbb{N}} X_{i}$ by:

$$
\begin{aligned}
\Theta: G \times X & \rightarrow X \\
(a, x) & \rightarrow \Theta(a, x) .
\end{aligned}
$$

For $k \geq 0$, is said to be $C^{\infty, k}$ I.L.H. if

(i) $\Theta$ induces a $C^{\infty}$ action:

$$
\Theta_{i}: G_{\imath+k} \times X_{i} \rightarrow X_{i} .
$$

(ii) $\Theta: G \times X \rightarrow X$ is a $C^{\infty}$ I.L.H. map. 
Notice that if the action of $G$ on $X$ is $C^{\infty, k}$ I.L.H., then for any a $\in G$, the map $x \rightarrow R_{a} x$ on $X$ is $C^{\infty}$ I.L.H. of order $\leq 0$.

We shall say that an I.L.H. action is free (resp. proper) if the $\Theta_{i}$ are free (resp. proper). We easily check that if the action $\Theta$ is free, we have for $(a, x) \in G \times X$, $\Theta(a, x)=x \Rightarrow a=e$. Let us check that if the action is proper, then for sequences $\left(a_{n}, x_{n}\right) \in G \times X$ such that $x_{n} \rightarrow x \in X$ and $\Theta\left(a_{n}, x_{n}\right) \rightarrow y \in X$, there is a subsequence $\left(a_{\phi(n)}\right)$ of $\left(a_{n}\right)$ that converges in $G$. The actions $\Theta_{i}$ being proper, for each $i \in \mathbb{N}$, there is a subsequence $\left(a_{\phi_{2}(n)}\right)$ of $\left(a_{n}\right)$ converging in $G_{i+k}$. Starting from $i=1$, we can iterate the extraction of a subsequence up to a certain $j$, thus obtaining a subsequence $\left(a_{\phi_{j}(n)}\right)$ of $\left(a_{n}\right)$ converging to $a_{j} \in \bigcap_{i=1}^{\jmath} G_{i+k}$. Using the diagonal principle, we can thus extract a subsequence $\left(a_{\phi(n)}\right)$ from $\left(a_{n}\right)$ which converges to an element $a \in G$.

Lemma 2.1. Let $G$ be an I.L.H. Lie group acting on the right (resp. left) through an action $\Theta$ on a $C^{\infty}$ I.L.H. variety $X=\bigcap_{i \in \mathbb{N}} X_{\imath}$ such that $G=\bigcap_{\imath \in \mathbb{N}} G_{i}$ where $G_{i}$ is a right (resp. left) semi-Hilbert Lie group. Under the assumption that

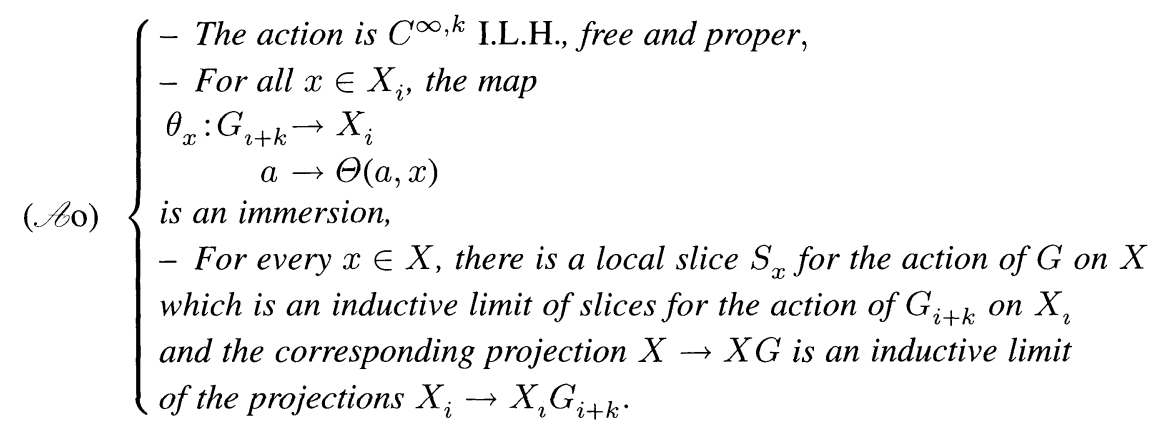

Then

1) $X / G$ is a $C^{\infty}$ I.L.H. manifold.

2) $X \rightarrow X / G$ is a principal fibre bundle with structure group $G$.

Proof. Since the action $\Theta$ is $C^{\infty, k}$ I.L.H. free and proper, for any $i$ the induced action $\Theta_{\imath}: G_{i+k} \times X_{i} \rightarrow X_{i}$ is $C^{\infty}$, free and proper (see the above definition). Since $\theta_{x}$ is an immersion, from Lemma 1.1 follows that $X_{i} / G_{i+k}$ is a $C^{\infty}$ Hilbert manifold and that $\pi_{\imath}: X_{i} \rightarrow X_{i} / G_{i+k}$ is a $C^{\infty}$ principal fibre bundle. In particular, for $x, y \in X$, setting $S_{x}=\bigcap_{i} S_{x, i}, S_{y}=\bigcap_{i} S_{y, i}$, the inductive families of local homeomorphisms $w_{x, i}: S_{x, i} \rightarrow \stackrel{\imath}{X}_{\imath} / G_{i+k}$ and $\stackrel{i}{\psi}_{y, \imath}: S_{y, i} \rightarrow X_{i} / G_{i+k}$ induce a family of $C^{\infty}$ transition mappings $\psi_{y, 2}^{-1} \circ \psi_{x, 2}$, the inductive limit of which yields a $C^{\infty}$ I.L.H. transition mapping for the quotient $X / G$. The quotient $X / G$ is therefore a $C^{\infty}$ I.L.H. manifold. In the same way, since the canonical projection $\pi$ is an inductive limit of a family of canonical projections $\pi_{i}$ local trivialisations $\phi: \pi^{-1}(U) \subset X \rightarrow U \subset X / G \times G$, $\phi(x)=\left(\pi(x), \tilde{\phi}_{x}(x)\right.$ of the bundle $X \rightarrow X / G$ can be obtained as inductive limits of families of local trivialisations $\phi_{i}: \pi_{\imath}^{-1}\left(U_{i}\right) \subset X_{i} \rightarrow U_{i} \subset X_{i} / G_{\imath+k} \times G_{\imath+k}$, $\phi_{i}(x)=\left(\pi_{i}(x), \tilde{\phi}_{i, x}(x)\right)$. Taking two open subsets $U=\bigcap_{\imath} U_{i}, V=\bigcap_{\imath} V_{\imath}$ of $X / G$, and two families of local trivialisations $\phi_{i}, \psi_{i}, i \in \mathbb{N}$ associated to these, the corresponding transition functions $x \rightarrow \tilde{\phi}_{i, x} \circ \tilde{\psi}_{i, x}^{-1}$ from $U_{\imath} \cap V_{\imath}$ to $G_{i+k}$ yield an inductive family 
of $C^{\infty}$ maps, the inductive limit of which is a $C^{\infty}$ I.L.H. transition function for the bundle $X \rightarrow X / G$. The canonical projection map $\pi: X \rightarrow X / G$ therefore yields a principal I.L.H. fibre bundle.

We now apply this lemma to the case of infinite dimensional manifolds modelled on spaces of sections of some vector bundle on a compact surface.

Theorem 2.2. Let $\mathscr{E}, \mathscr{H}, \mathscr{H}$ be $C^{\infty}$ vector fibre bundles on $M$ (of dimension $n$ ) with finite dimensional fibres built up from tensor bundles on $M$. Let $X=\bigcap_{i} X_{\imath}$ be a $C^{\infty}$ I.L.H. variety modelled on $C^{\infty}(\mathscr{E})$ such that each $X_{i}$ is modelled on $H^{i}(\mathscr{E})$ and is equipped with a smooth $L^{2}$ riemannian structure which yields a $C^{\infty}$ exponential map with the usual properties (in particular, it should induce a $C^{\infty}$ local diffeomorphism from $T X$ to $X)$.

Let $G=\bigcap_{i \in \mathbb{N}} G_{\imath}$ be an I.L.H. Lie group which is a semi-direct product of two groups, where $G=H \odot K, H=\bigcap_{i \in \mathbb{N}} H_{\imath}$ and $K=\bigcap_{i \in \mathbb{N}} K_{i}$ are I.L.H. manifolds modelled on $C^{\infty}(\mathscr{H})$ and $C^{\infty}(\mathscr{K})$ respectively. $G$ acts on $X$ through a right action $\Theta$, such that for $\left(a=\left(a^{\prime}, a^{\prime \prime}\right), x\right) \in G \times X, \Theta(a, x)=R_{a}^{\prime}\left(R_{a^{\prime \prime}} x\right), H$ acting on $X$ through isometries. We shall assume as before that condition (1.3) is fulfilled, namely for $a=\left(a^{\prime}, a^{\prime \prime}\right) \in G$ :

$$
D_{a} \theta_{x}=R_{a^{\prime}}^{*} \tau_{R_{a^{\prime \prime} x}} \circ D R_{a}^{-1} .
$$

We furthermore assume that the $L^{2}$ weak riemannian structure on $X_{\imath}$ is $H$-invariant and that the map

$$
\begin{aligned}
G_{i+k} & \rightarrow T_{a} G_{i+k} \\
a & \rightarrow\left(D_{a} \theta_{x}\right)^{*}
\end{aligned}
$$

is smooth. Here $\left(D_{a} \theta_{x}\right)^{*}$ denotes the adjoint of $D_{a} \theta_{x}$ w.r.t. to an $L^{2}$ weak riemannian structure on $G$. Under the following assumption:

$$
\text { (.A) }\left\{\begin{array}{l}
- \text { The action } \Theta \text { is } C^{\infty, k} \text { I.L.H. free and proper, } \\
- \text { For any } x \in X \text {, the map } \tau_{x}: C^{\infty}(\mathscr{F}) \rightarrow C^{\infty}(\mathscr{E}) \\
\text { is an injective differential operator with injective symbol. }
\end{array}\right.
$$

Then

1) $X / G$ is a $C^{\infty}$ I.L.H. manifold.

2) The projection $\pi: X \rightarrow X / G$ induces a $C^{\infty}$ I.L.H. principal fibre bundle structure.

Remarks. 1) In applications, the assumption on the existence of an exponential map associated to the weak $L^{2}$ riemannian structure with the usual properties arises as a consequence of the general features of the manifold $X$ and the way such a riemannian metric is built on $X$. Indeed, taking for example $\mathscr{E}$ to be a tensor bundle on $M$, then each $X_{\imath}$ modelled on $H^{\imath}(\mathscr{E})$ can be equipped with a natural smooth $L^{2}$ riemannian structure $\langle\cdot, \cdot\rangle_{L^{2}}$ induced at point $x \in X_{i}$ by a bilinear form:

$$
\begin{aligned}
H^{i}(\mathscr{E}) \times H^{i}(\mathscr{E}) & \rightarrow \mathbb{R} \\
\left(t, t^{\prime}\right) & \rightarrow \int_{M} \sqrt{g}_{x}(\alpha)\left(t, t^{\prime}\right)_{x}(\alpha) d \alpha,
\end{aligned}
$$

where $g_{x}$ is a riemannian metric on $M$ of class $H^{i}$ and $(\cdot, \cdot)_{x}$ a scalar product on the fibre of the bundle $\mathscr{E}$ induced by the metric $g_{x}$ by contraction of tensors. Applying to 
this weak riemannian metric the usual construction of a riemannian connection, one obtains for $U, V \in H^{s}(\mathscr{E})$ an $L^{2}$ field $\nabla_{U} V$. Using the fact that $\nabla_{U} V$ is characterised by

$$
\begin{aligned}
\nabla_{U} V-\nabla_{V} U & =[U, V], \\
W\langle U, V\rangle_{L^{2}} & =\left\langle D_{W} U, V\right\rangle_{L^{2}}+\left\langle U, D_{W} V\right\rangle_{L^{2}},
\end{aligned}
$$

one can show that since the riemannian metric is built up from tensors on $M$ of class $H^{i}$ (arising from the riemannian metric $g_{x}$ ), $\nabla_{U} V$ is in fact an $H^{i}$ field so that $\nabla$ is a riemannian connection in the usual sense and the smoothness of the riemannian metric on $X_{\imath}$ induces the smoothness of the connection. From this smooth connection one can build an exponential map with the usual properties.

2) The smoothness of the map $a \rightarrow D_{a} \theta_{x}^{*}$ also appears in the applications as a natural consequence of the general features of the manifold $X$ and the group $G$ and the associated riemannian structures on them. Indeed, if the vector bundles involved in the structure of $X$ and $G$ are tensor bundles, the smoothness of the map $a \rightarrow\left(D_{a} \theta_{x}\right)^{*}$ then appears as a consequence of the smoothness of the map $a \rightarrow D_{a} \theta_{x}$ and the features of the scalar products involved in the construction of the adjoint operator.

Proof of Theorem 2.2. We shall do the proof in the simpler case $G=H$, for which assumption (1.3) is of course trivially satisfied, pointing out the required modifications for the general case $G \neq H$. Indeed, the general case can be proved in a similar way, using assumption (1.3) and the $H$-invariance of the metric.

As in the proof of Theorem 1.2, using the relation between $D_{a} \theta_{x}$ and $\tau_{x}$, we easily check that the second condition in $\left(f_{0}\right)$ is satisfied since $\tau_{x}$ is injective and has injective symbol. Since the group $G$ acts on each $X_{i}$ (because $G \subset G_{i+k}$ and $G_{i+k}$ acts on $X_{i}$ since the action is $C^{\infty, k}$ ), the canonical projection $\pi: X \rightarrow X / G$ is an inductive limit of canonical projections $\pi_{i}: X_{\imath} \rightarrow X_{\imath} / G_{i+k}$. The only thing left to be checked in order to apply Lemma 2.1 is the existence, at each point $x \in X$ of a local slice $S_{x}$ obtained as inductive limit of a family of local slices $S_{x, \imath}$ for the action of $G_{i+k}$ on $X_{i}$.

We shall build the slice along the lines of the construction which can be found in $[\mathrm{E}]$ in the case of the group of diffeomorphisms acting on the manifold of riemannian metrics. We shall closely follow the scheme of the construction of a slice done in the proof of Lemma 1.1, replacing here the strong riemannian structure on the Hilbert manifold $X_{\imath}$ underlying that construction by the weak $L^{2}$ riemannian structure common to all the $X_{\imath}, i \in \mathbb{N}$. Indeed, if for $x \in X$, one builds a slice $S_{x, \imath}$ for the action of $G_{i+k}$ on each $X_{i}$ using the strong riemannian structure on $X_{i}$, as in the proof of Lemma 1.1, the inductive limit $\bigcap_{i} S_{x, \imath}$ might well reduce to the point $x$. Replacing here the strong riemannian structure on the Hilbert manifold $X_{\imath}$ underlying that construction by a weak $L^{2}$ riemannian structure common to all the $X_{i}, i \in \mathbb{N}$, we shall obtain a family of "comparable slices," the inductive limit of which will be a slice for the action of $G$ on $X$.

Set for $x \in X, i \in \mathbb{N}$ :

$$
N\left(O_{x, \imath}\right) \equiv\left\{v \in T X_{i} / O_{x, \imath} \text {, such that }\langle v, w\rangle_{L^{2}}=0, \forall w \in T O_{x, \imath}\right\},
$$

where $O_{x, i}$ is the orbit of $x$ under the action of $G_{i+k}$ and $\langle\cdot, \cdot\rangle_{L^{2}}$ is the scalar product on $T X_{i}$ induced by the $L^{2}$ riemannian structure on $X_{i}$. Since this $L^{2}$ riemannian structure is weak, this bundle is not an ordinary normal subbundle to $T O_{x, i} / O_{x, \imath}$ in 
$T X_{\imath} / O_{x, \imath}$. To see that it is all the same a $C^{\infty}$ subbundle of $T X_{\imath} / O_{x, \imath}$, we shall identify it to the kernel of a surjective $C^{\infty}$ fibre bundle map.

Since for $a \in G_{\imath+k}$, we have $T_{R_{a} x} O_{x, \imath}=\operatorname{Im} D_{a} \theta_{x}$, the fibre of $N\left(O_{x, i}\right)$ at point $R_{a} x$, is $\left(\operatorname{Im} D_{a} \theta_{x}\right)^{\perp}=\operatorname{Ker}\left(D_{a} \theta_{x}\right)^{*}$. Let us introduce a fibre bundle map $P: T X_{\imath} / O_{x, \imath} \rightarrow T O_{x, i}$ defined on the fibre at $R_{a} x$ by $D_{a} \theta_{x} \circ\left(D_{a} \theta_{x}^{*} \circ D_{a} \theta_{x}\right)^{-1} \circ D_{a} \theta_{x}^{*}$ and let us describe its image. Since $\tau_{x}$ has injective principal symbol, there is an orthogonal splitting for the $L^{2}$ scalar product $T_{x} X_{\imath}=\operatorname{Im} \tau_{x} \oplus \operatorname{Ker} \tau_{x}^{*}$. But since $D_{a} \theta_{x}=R_{a}^{*} \tau_{x} D R_{a}^{-1}$ (resp. $D_{a} \theta_{x}=R_{a^{\prime}}^{*} \tau_{R_{a^{\prime \prime}} x} D R_{a}^{-1}$ if $G=\stackrel{x}{H} \odot K, H \neq G$ ), using the $G$-invariance (resp. $H$ invariance if $H \neq G$ ) of the $L^{2}$ riemannian structure, we see that $T_{R_{a} x} X_{\imath}$ also splits into an orthogonal direct $\operatorname{sum} T_{R_{a} x} X_{i}=\operatorname{Im} D_{a} \theta_{x} \oplus \operatorname{Ker} D_{a} \theta_{x}^{*}$. From this easily follows that $\operatorname{Im} D_{a} \theta_{x}=\operatorname{Im} D_{a} \theta_{x} \circ\left(D_{a} \theta_{x}^{*} \circ D_{a} \theta_{x}\right)^{-1} \circ D_{a} \theta_{x}^{*}$ (the inclusion from right to left is of course trivial), so that $P$ is a surjective operator since the fibre of $T O_{x, i}$ at point $R_{a} x$ is $\operatorname{Im} D_{a} \theta_{x}$. In the same way, the above orthogonal splitting yields $\operatorname{Ker}\left(D_{a} \theta_{x}\right)^{*}=\operatorname{Ker} D_{a} \theta_{x} \circ\left(D_{a} \theta_{x}^{*} \circ D_{a} \theta_{x}\right)^{-1} \circ D_{a} \theta_{x}^{*}$ (here the inclusion from left to right is of course trivial), so that the kernel of $P$ at the fibre above $R_{a} x$ is indeed $\operatorname{Ker}\left(D_{a} \theta_{x}\right)^{*}$ which coincides with the fibre of $N\left(O_{x, \imath}\right)$ at point $R_{a} x$.

Let us now check that this fibre bundle map is smooth. Since the right action of $G_{i+k}$ on $X_{\imath}$ is smooth, this boils down to checking the smoothness of the maps $a \rightarrow D_{a} \theta_{x}$ and $a \rightarrow\left(D_{a} \theta_{x}\right)^{*}$. Again by the smoothness of the action of $G_{\imath+k}$ on $X_{i}$, the first of these two is smooth. Using the assumption made on the latter, we conclude that the map $P$ is a smooth fibre bundle map. Hence its kernel $N\left(O_{x, i}\right)$ is a smooth fibre bundle.

Using the exponential map associated to this weak riemannian structure, we can build as in the proof of Lemma 1.1 a family of slices $S_{x, i}=\exp \{v \in$ $\left.N_{x}\left(O_{x, i}\right),\|v\|_{i}<\varepsilon\right\}$ for the action of $G_{\imath+k}$ on $X_{i}$, with $\varepsilon$ chosen small enough. Here $\|\cdot\|_{i}$ is the norm associated to the Hilbert structure on $X_{i}$.

Let us check that the inductive limit of these slices $S_{x}=\cap S_{x, i}$ yields a slice for the action of $G$ on $X$. It is clear that for $a \in G, R_{a} S_{x} \subset S_{x}$ since $R_{a} S_{s, i} \subset S_{x, i}$ for all $i$. Assume $R_{a} S_{x} \cap S_{x} \neq \phi$, then, by construction of $S_{x}$, for every $i, R_{a} S_{x, \imath} \cap S_{x, \imath} \neq \phi$ so that $a=e$ since the $S_{x, \imath}$ are slices. Since for every $i, U_{\imath} \times S_{x, i} \simeq V_{x, i}$ for some $U_{i} \in G_{i+k}$ open neighborhood of $e, V_{x, i}$ open neighbourhood of $x$ in $X_{i}$, the diffeomorphism being $C^{\infty}$, there is a $C^{\infty}$ I.L.H. diffeomorphism $U \times S_{x} \simeq V_{x}$ in the limit, where $U=\cap_{\imath} U_{i}, V_{x}=\cap_{i} V_{x, i}$ are open neighborhoods in $G$ and $X$ respectively, containing $e$ and $x$ respectively. Thus $S_{x}$ defines a slice for the action of $G$ on $X$.

Example. On can apply this theorem to the action of $G=\mathscr{D}_{0}(M) \odot \mathscr{W}(M)$ on $X=\mathscr{M}(M)$, where these spaces are defined as before, the underlying manifold $M$ being of dimension 2. Here $H=\mathscr{D}_{0}(M), K=\mathscr{W}(M)$. Let us check that the hypotheses of the above theorem are satisfied. The action is $C^{\infty, 1}$, free and proper and the operator $\tau_{x}$ is injective and has injective symbol as can be seen from the example in Sect. I. Moreover, there is a $\mathscr{D}^{s+1}(\Lambda)$ invariant weak $L^{2}$ riemannian metric on $\mathscr{C ^ { s }}(\Lambda)$ given by the scalar product at point $g$ defined as $\langle h, k\rangle_{g}=\int \sqrt{g}(\eta) h(\eta) k(\eta) d \eta$. It induces a $C^{\infty}$ exponential map which is a local $C^{\infty}$ diffeomorphism $T \mathscr{M}^{s} \rightarrow \mathscr{M}^{s}$ ([E] par. 4). Furthermore, it can be shown that the map $\left(f, e^{\phi}\right) \rightarrow\left(D_{\left(f, e^{\phi}\right)} \theta_{g}\right)^{*}$ is smooth; this follows from the fact shown in [E] (p. 31) that the map $f \rightarrow\left(D_{f} \theta_{g}\right)^{*}$ is smooth and the fact that $D_{\left(f, e^{\phi}\right)} \theta_{g}=D_{f} \theta_{e^{\phi}}$. Hence all the assumptions of the theorem are satisfied and $\mathscr{C}(M) / \mathscr{D}_{0}(M) \odot \mathscr{W}(M)$ is a $C^{\infty}$ I.L.H. manifold and the canonical projection of $\mathscr{C}(M)$ onto this quotient yields a principal fibre bundle. 


\section{The Faddeev Popov Determinant for Gauge Theories}

Let $G=H \odot K$ be a right semi-Hilbert (resp. I.L.H.) Lie group acting on the right on a $C^{\infty}$ Hilbert (resp. I.L.H.) manifold, where $H$ and $K$ are semi-Hilbert (resp. I.L.H.) Lie goups. Let us assume that $H, K$ and $X$ are modelled on spaces of Sobolev (resp. $C^{\infty}$ ) sections of smooth vector bundles $\mathscr{H}, \mathscr{K}$ and $\mathscr{E}$ respectively with finite dimensional fibres on a fixed smooth compact Riemannian manifold $M$ without boundary.

$X$ is equipped with an $H$-invariant $L^{2}$ riemannian structure induced by the riemannian metric on $M$ (resp. which induces an exponential map with the usual properties). As before, we shall identify for $a=\left(a^{\prime}, a^{\prime \prime}\right)$, the tangent spaces $T_{a} G=T_{a^{\prime}} H \times T_{a^{\prime \prime}} K$ and shall furthermore assume (see formula (1.3)) that for $a=\left(a^{\prime}, a^{\prime \prime}\right) \in G, u^{\prime} \in H, u^{\prime \prime} \in K$, we have:

$$
D_{a} \theta_{x}=R_{a^{\prime}}^{*} \tau_{R_{a^{\prime \prime}}} D R_{a}^{-1} .
$$

In the former sections, we showed that if the action $\Theta$ of $G$ on $X$ is smooth (resp. $C^{\infty, k}$ I.L.H.), free and proper and if moreover, for all $x \in X$, the operator

$$
\tau_{x}: T_{e} G \rightarrow T_{x} X
$$

is an injective differential operator with injective symbol (resp. and if the map $a \rightarrow\left(D_{a} \theta_{x}\right)^{*}$ is smooth), then $X / G$ has a smooth manifold structure (possibly infinite dimensional) and $X \rightarrow X / G$ a smooth principal fibre bundle structure.

In this present section, we show that these same hypotheses yield the existence of the Faddeev-Popov determinant.

Let us assume conditions (A) of Theorem 1.2 (resp. ( $\mathscr{b})$ of Theorem 2.2) are satisfied. Theorem 1.2 (resp. Theorem 2.2) tell us that the canonical projection $\pi: X \rightarrow$ $X / G$ is a principal fibre bundle. For $x \in X$, let $\Sigma_{x}$ be a local slice of this bundle. For $a=\left(a^{\prime}, a^{\prime \prime}\right) \in G$, the Faddeev-Popov operator $F_{a, x}^{\Sigma_{x}}$ reads:

$$
\begin{aligned}
F_{a, x}^{\Sigma_{x}}: T_{e} G \times T_{x} \Sigma_{x} & \rightarrow T_{R_{a} x} X \\
\left(\left(u^{\prime}, u^{\prime \prime}\right), h\right) \rightarrow & D_{a} \theta_{x}\left(D R_{a^{\prime}} u^{\prime}+D R_{a^{\prime \prime}} u^{\prime \prime}\right)+R_{a}^{*} h \\
& =R_{a}^{*} \tau_{R_{a^{\prime \prime}}} u+R_{a}^{*} h,
\end{aligned}
$$

where $u=u^{\prime}+u^{\prime \prime} \in T_{e} H \times T_{e} K$.

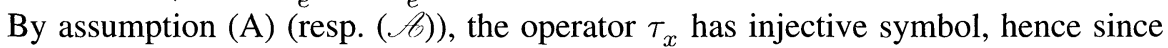
the manifold $M$ is compact, we have an orthogonal splitting w.r.t. the scalar product induced by the riemannian structure on $X$ :

$$
T_{T_{a} x} X=R_{a^{\prime}}^{*}\left(\operatorname{Im} \tau_{R_{a^{\prime \prime}}} \oplus \operatorname{Ker} \tau_{R_{a^{\prime \prime}} x}^{*}\right) .
$$

Thus, if $\pi_{x}$ denotes the orthogonal projection onto $\operatorname{Im} \tau_{x}$ which is well defined since the image is closed, the operator $F_{a, x}^{\Sigma_{x}}$ seen as an operator from $T_{a} G \times T_{x} \Sigma_{x}$ to $R_{a^{\prime}}^{*}\left(\operatorname{Im} \tau_{R_{a^{\prime \prime}} x}\right) \times R_{a^{\prime}}^{*} \operatorname{Ker} \tau_{R_{a^{\prime \prime}} x}$ can be written as a matrix operator:

$$
F_{a, x}^{\Sigma_{x}}=R_{a^{\prime}}^{*}\left[\begin{array}{cc}
\tau_{R_{a^{\prime \prime} x}} & \pi_{R_{a^{\prime \prime} x}}\left(R_{a^{\prime \prime}}^{*} \cdot\right) \\
0 & \left(11-\pi_{R_{a^{\prime \prime}} x}\right)\left(R_{a^{\prime \prime}}^{*} \cdot\right)
\end{array}\right] .
$$

Notice that if $\Sigma_{x}$ is an orthogonal slice to the orbits, this matrix operator takes the simple diagonal form $R_{a^{\prime}}^{*}\left[\begin{array}{cc}\tau_{R_{a^{\prime \prime}} x} & 0 \\ 0 & \mathbb{1}\end{array}\right]$, since $\pi_{x}\left(T_{x} \Sigma_{x}\right)=\{0\}$. 
We now define the determinant of $F_{a, x}^{\Sigma_{x}}$ in two cases, namely first when the slice is orthogonal to the orbit (which is the case for Yang-Mills theory) the quotient $X / G$ being possibly infinite dimensional, and then when the slice is arbitrary but the quotient is finite dimensional. For this we shall use the notion of heat-kernel regularised determinants for strictly positive elliptic operators on smooth boundaryless compact manifolds. As pointed out in Appendix A.2, the heat-kernel regularising methods discussed for example in [AJPS] and [Pa] in the context of string theory, extend to elliptic operators with $H^{k}$ coefficients, for $k$ large enough, since these operators have the same spectral properties as the ones with $C^{\infty}$ coefficients.

When $A$ is a strictly positive elliptic operator on a vector fibre bundle $\mathscr{V}$ on the smooth compact boundaryless surface $M$, this vector bundle being equipped with a scalar product, we denote by $\operatorname{det} A$ its regularised determinant. We can extend this notion of determinant to injective differential operators from a vector bundle $\xi$ to a vector bundle $\eta$ with injective symbol, setting for such an operator $B$, $\operatorname{det} B=\sqrt{\operatorname{det}\left(B^{*} B\right)}$, where $B^{*}$ is the formal adjoint to $B$. Under assumption $(A)$ of Theorem 2.2, we can therefore define $\operatorname{det} \tau_{x}$ as the determinant of the injective differential operator $\tau_{x}$ with injective symbol.

In the first case, i.e. when the slice is orthogonal to the orbit, it is clear from the shape of the matrix in (3.3) that the determinant of the Faddeev-Popov operator (3.1) can be defined as the regularised determinant of the injective differential operator with injective symbol $\tau_{R_{a^{\prime \prime}} x}$. The pull-back $R_{a^{\prime}}$ in front of the matrix does not have any influence since the $L^{2}$-Riemannian structure on $X$ is $H$ invariant.

The second case, i.e. when the quotient $X / G$ is finite dimensional and the slice arbitrary, has been studied in a former paper [Pa] in which it was shown (see Proposition 3.1) that the determinant of the Faddeev-Popov operator is up to a multiplicative factor (the determinant of a finite dimensional matrix which depends on the choice of the slice), the regularised determinant of $\tau_{R_{a^{\prime \prime}}}$.

Let us summarise the above discussion in the following theorem which concerns both the Hilbert and the I.L.H. case.

Theorem 3.1. Let $G=H \odot K$ be a semi-Hilbert (resp. I.L.H.) Lie group acting on the right on a $C^{\infty}$ Hilbert (resp. I.L.H.) manifold, where $H$ and $K$ are semi-Hilbert (resp. I.L.H.) Lie groups. Let us assume that $H, K$ and $X$ are modelled on spaces of Sobolev (resp. $C^{\infty}$ ) sections of smooth vector bundles $\mathscr{H}, \mathscr{K}$ and $\mathscr{E}$ respectively with finite dimensional fibres on a fixed smooth compact Riemannian manifold $M$ without boundary. $X$ (resp. $X$, where $X=\cap_{i} X_{i}$ ) is (resp. are) equipped with an $H$-invariant $L^{2}$ Riemannian structure induced by the riemannian metric on $M$ (resp. and we shall assume that there is an exponential map induced by this $L^{2}$ structure with the usual properties, namely such that it induces a local $C^{\infty}$ diffeomorphism exp :TX $X_{i} \rightarrow X_{i}$ for each i). Under the assumption that

- The action of $X$ on $G$ is $C^{\infty}$ (resp. $C^{\infty, k}$ I.L.H.), free and proper,

- $\forall x \in X$, the operator

$$
\tau_{x}: T_{e} G \rightarrow T_{x} X
$$

is an injective differential operator with injective symbol (resp. and the map $a \rightarrow D_{a} \theta_{x}^{*}$ is smooth).

Then

1) The quotient $X / G$ has a smooth manifold structure (possibly an infinite dimensional Hilbert (resp. I.L.H.) structure) and the canonical projection $\pi: X \rightarrow X / G$ yields a principal fibre bundle structure. 
2) Let $\Sigma_{x}$ be a local slice for this bundle at point $x \in X$. If either this slice is orthogonal to the orbit of $x$, or - when the quotient $X / G$ is finite dimensional - for an arbitrary local slice $\Sigma_{x}$, the Faddeev-Popov operator $F_{a, x}^{\Sigma_{x}}$ (where $a \in G$ ) has a well defined determinant:

$$
\operatorname{det} F_{a, x}^{\Sigma_{x}}=\operatorname{det}\left(\tau_{R_{a^{\prime \prime}}}\right) \operatorname{det}\left(\mathbb{1}-\pi_{R_{a^{\prime \prime}} x} / T_{x} \Sigma_{x}\right),
$$

where this last determinant is defined as 1 when the slice $\Sigma_{x}$ is orthogonal to the orbit at $x$ and as the determinant of a finite dimensional operator when the quotient $X / G$ (and hence when $\Sigma_{x}$ ) is finite dimensional.

\section{Applications}

Application to Yang-Mills Theory. This corresponds to the first case when the local slice is chosen orthogonal to the orbit. The quotient space here is infinite dimensional. On the other hand, we saw that in the case of Yang-Mills theory, $G=H$.

We recall that in the case of Yang-Mills theory, $X=\overline{\mathscr{A}}_{s}, G=G_{s+1}$. For $A \in \overline{\mathscr{A}}_{s}$, we can choose a local slice contained in $\Sigma_{A}=\left\{A+h, h \in H^{s}\left(E_{\text {ad }} \otimes T^{*} M\right), d_{A}^{*} h=\right.$ $0\}$ orthogonal to the orbit $A$. For $g \in G_{s+1}, A \in \mathscr{A}_{s}$, the Faddeev-Popov operator reads (see e.g. $[\mathrm{Ja}]$ )

$$
F_{g, A}^{\Sigma_{A}}=R_{g}^{*}\left[\begin{array}{cc}
\tau_{A} & 0 \\
0 & 11
\end{array}\right]
$$

where the operator $\tau_{A}$ is given for $A \in \mathscr{C}_{s}$, by $d_{A}$, the covariant derivative associated to the connection $A$. In the example concerning Yang-Mills theory of Sect. I, we saw that $\tau_{A}=d_{A}$ is an injective differential operator with injective symbol with coefficients of Sobolev class, namely for $s>\frac{n}{2}+3, d_{A} \in \operatorname{Inj}^{s-1}\left(E_{\mathrm{ad}}, E_{\mathrm{ad}} \otimes T^{*} M\right)$. From the results of Appendix A.2, we can define the regularised determinant of $d_{A}$ and we have for $g \in G_{k+1}$ :

$$
\operatorname{det} F_{g, A}^{\Sigma_{A}}=\operatorname{det} d_{A}
$$

Application to Bosonic Closed Strings. This corresponds to the second case, when the quotient is finite dimensional and the local slice arbitrary. We recall that here, the group $H$ corresponds to $\mathscr{D}_{0}(M)$, the group $G$ to $\mathscr{D}_{0}(M) \cdot \mathscr{W}(M)$.

If the genus $p$ of $M$ is strictly larger than 1 , one can show the existence of a global section $\Sigma$ of the bundle $\mathscr{C}(M) \rightarrow \mathscr{L}(M) / \mathscr{D}_{0}(M) \odot \mathscr{W}(M)$ given by the harmonic gauge (see e.g. [AHKPS]). This section is of the form $\Sigma \equiv\left\{g_{t}, t \in \mathscr{T}(M)\right\}$, where $\mathscr{T}(M)$ is the Teichmüller space on $M$ and where $g_{t}$ varies in the riemannian metrics of curvature -1 . One finds for the Faddeev-Popov determinant (see $[\mathrm{Pa}]$ ):

$$
\operatorname{det} F_{f^{*} e^{\phi} g}^{\Sigma}=\operatorname{det} F_{\bar{g}}^{\Sigma}=\operatorname{det} P_{\bar{g}} \frac{\operatorname{det}\left(\left\langle\Psi_{\bar{g}}^{\imath}, \chi_{\bar{g}}^{\jmath}\right\rangle\right)_{\bar{g}}}{\operatorname{det}\left(\left\langle\psi_{\bar{g}}^{\imath} \psi_{\bar{g}}^{i}\right\rangle_{g}\right)_{\bar{g}}}
$$

where $\bar{g}=e^{\phi} g$ and $\psi_{g}^{i}, i=1, \ldots, 6 p-6$ is a basis of $\operatorname{Ker} P_{g}^{*}, \chi_{g}^{i}, i=1, \ldots, 6 p-6$ being a basis of $T_{g} \Sigma$, whenever $g \in \Sigma$. 


\section{Appendix A.1: Proof of Theorem 1.2}

Step 1. The action being proper, for $x \in X, \theta_{x}$ is a closed mapping and hence a homeomorphism onto its image. Being both an injective immersion and a homeomorphism onto its image, it is a diffeomorphism onto its image (by the inverse mapping theorem). Hence $G \simeq O_{x}$, the orbit of $x$ is a closed submanifold of $X$.

Step 2. Since $X$ is a Hilbert manifold, there is a $C^{\infty}$ strong riemannian structure on $X$ and a corresponding $C^{\infty}$ exponential map exp $: T X \rightarrow Y X$. Let us define

$$
N\left(O_{x}\right) \equiv\left\{v \in T X / O_{x}, \text { such that }\langle v, w\rangle=0, \forall w \in T O_{x}\right\},
$$

which is a $C^{\infty}$ subbundle of $T X / O_{x}$. Set $N_{x}\left(O_{x}\right) \equiv N\left(O_{x}\right) \cap T_{x} X$ and

$$
N_{\varepsilon, x} \equiv\left\{v \in N_{x}\left(O_{x}\right),\|v\|<\varepsilon\right\} .
$$

For $\varepsilon$ small enough, the exponential map exp is a diffeomorphism on $N_{\varepsilon, x}$ and we set

$$
S_{\varepsilon, x} \equiv \exp \left(N_{\varepsilon, x}\right)
$$

which is a $C^{\infty}$ submanifold of $X$ with tangent space $\left(T_{x} O_{x}\right)^{\perp}$.

Step 3. $S_{\varepsilon, x}$ has the properties of a slice, namely

(a) $R_{a} S_{\varepsilon, x} \cap S_{\varepsilon, x} \neq \phi \Rightarrow a=e$,

b) $\forall x \in X$, there is a neighbourhood $U$ of $e$ in $G$, a neighbourhood $V_{x}$ of $x$ in $X$ such that

$$
U \times S_{\varepsilon, x} \simeq V_{x}
$$

The assertion a) follows from the fact that the action is continuous, proper and free. Assuming the assertion a) does not hold, letting $\varepsilon$ take the values $\frac{1}{n}$, one can build sequences $\left(a_{n}\right), a_{n} \in G$ and $\left(x_{n}\right), x_{n} \in X$ such that $x_{n} \in S_{\varepsilon, x}$ and $R_{a_{n}} x_{n} \in S_{\varepsilon, x}$. By construction, we have that $x_{n} \rightarrow x$ and $R_{a_{n}} x_{n} \rightarrow x$. The properness of the action yields the existence of a subsequence $\left(a_{\phi(n)}\right)$ of $\left(a_{n}\right)$ converging to some $a \in G$, from which would follow, by the smoothness of the action that $R_{a_{\phi(n)}} x_{\phi(n)}$ tends to $R_{a} x$. But then, in the limit we would have $R_{a} x=x$, which yields $a=e$ since the action is free.

The assertion $\mathrm{b}$ ) follows from the fact that the action is $C^{\infty}$ and that $\theta_{x}$ is an immersion combined with the local inversion theorem. The tangent map at point $(e, x)$ to

$$
\begin{aligned}
F: G \times X & \rightarrow X \\
(a, x) & \rightarrow R_{a} x
\end{aligned}
$$

reads

$$
\begin{aligned}
D_{e, x} F: T_{e} G \times T_{x} S_{\varepsilon, x} & \rightarrow T_{R_{a} x} X \\
(v, w) & \rightarrow \tau_{x} v+w,
\end{aligned}
$$

where $\tau_{x} \equiv D_{e} \theta_{x}$ is the tangent map to $\theta_{x}$ at point $e \in G$. The injectivity of $D_{e, x} F$ is a consequence of that of $\tau_{x}$ and of the equality $T_{x} S_{\varepsilon, x}=\left(\operatorname{Im} \tau_{x}\right)^{\perp}$. The fact that its range is closed follows from the fact that the range of $\tau_{x}$ is closed. The local inverse function theorem then yields the existence of a neighborhood $U$ of $e \in G$, a real number $\varepsilon>0$ and a neighborhood $V_{\varepsilon, x}$ of $x$ small enough such that

$$
F: U \times S_{\varepsilon, x} \rightarrow V_{\varepsilon, x}
$$

be a $C^{\infty}$ diffeomorphism. 


\section{Appendix A.2: Differential Operators with Coefficients in $\boldsymbol{H}^{m}$}

This Appendix is a review of some properties of elliptic operators and of differential operators with injective symbol that have coefficients in a Sobolev space $H^{m}$, for $m$ large enough.

Let $M$ be a smooth compact Riemannian manifold without boundary of finite dimension. Let $\xi$ and $\eta$ be two vector bundles on $M$ with finite dimensional fibres, chosen in such a way that they naturally inherit a euclidean structure from the Riemannian structure on $M$. Typically, we shall choose bundles such as the trivial bundle $M \times \mathbb{R}$, tensor bundles $T M^{k} \otimes T^{*} M^{l}$, or bundles built up from an additional principal fibre bundle $\mathscr{P}$ on $M$ with structure group $\mathscr{G}$ like $\mathscr{P} \times_{\mathscr{G}} \mathbf{g}, \mathscr{P} \times_{\xi} \mathbf{g} \otimes T^{*} M$, where $\mathscr{G}$ is a compact semi-simple matrix Lie group and $\mathbf{g}$ the Lie algebra of $\mathscr{G}$.

Let $C^{k}(\xi)$ (resp. $C^{k}(\eta)$ ) denote the space of sections of $\xi$ (resp. $\eta$ ) of classes $C^{k}$ and $C^{\infty}(\xi)$ (resp. $C^{\infty}(\eta)$ ), the space of $C^{\infty}$ sections of $\xi$ (resp. $\eta$ ). Let $L^{2}(\xi)$ and $H^{k}(\xi)$ (resp. $L^{2}(\eta)$ and $\left.H^{k}(\eta)\right)$ be the closure of $C^{\infty}(\xi)\left(\right.$ resp. $C^{\infty}(\eta)$ ) w.r.t. the $L^{2}$ and the $H^{k}$ Riemannian structures induced on $\xi$ (resp. $\eta$ ) by the Riemannian structure on $M$. The corresponding norms will be denoted by $\|\cdot\|_{L^{2}}$ and $\|\cdot\|_{k}$.

Let now $D: C^{\infty}(\xi) \rightarrow H^{m}(\eta)$ be a differential operator of order $k$ with injective symbol, locally expressed as

$$
D=\sum_{|\alpha| \leq k} a_{\alpha} \partial_{\alpha},
$$

where each $a_{\alpha} \in H^{m}, m>\sup \left\{\frac{n}{2}, k\right\}$. For $0 \leq s \leq m+k, D$ extends uniquely to a continuous linear operator $D_{s}: H^{s}(\xi) \rightarrow H^{s-k}(\eta)$ (see e.g. [KR] p. 57).

Let us introduce a class of elliptic operators

$$
\begin{aligned}
\operatorname{Ell}^{m}(\xi, \eta) \equiv & \left\{D: C^{\infty}(\xi) \rightarrow H^{m}(\eta), \text { s.t. } D=\sum a_{\alpha} D^{\alpha}\right. \text { is elliptic } \\
& \text { and with } \left.a_{\alpha} \in H^{m} \text {, s.t. } m>\frac{n}{2}+1\right\} .
\end{aligned}
$$

Garding's inequality extends to this class of elliptic operators (see e.g. [C] Theorem 3.6):

Theorem A.2. For $D \in \mathrm{Ell}^{m}(\xi, \eta)$ of order $k$,

$$
\|f\|_{k} \leq C\left(\|D f\|_{L^{2}}+\|f\|_{L^{2}}\right)
$$

for all $f \in L^{2}(\xi)$, such that $D f \in L^{2}(\eta)$.

For $D: C^{\infty}(\xi) \rightarrow H^{m}(\eta)$, we can define the formal adjoint $D^{*}$ defined on a subspace of $L^{2}(\eta)$. Let us introduce a class of differential operators with injective symbol

$$
\begin{aligned}
& \operatorname{Inj}^{m}(\xi, \eta) \equiv\left\{D: C^{\infty}(\xi) \rightarrow H^{m}(\eta), \text { s.t. } D^{*} D \in \operatorname{Ell}^{m-k}(\xi, \eta),\right. \\
&\text { where } k \text { is the order of } D\} .
\end{aligned}
$$

The Hodge Kodaira like decomposition theorem extends to these classes of elliptic operators and of differential operators with injective symbol (see e.g. [C] Theorem 3.12 and Theorem 3.13): 
Theorem A.2.2. If $D \in \operatorname{Ell}(\xi, \eta)$ (resp. $D \in \operatorname{Inj}^{m}(\xi, \eta)$ ) is an operator of order $k$ with $m>\frac{n}{2}+k\left(\right.$ resp. $\left.m>\frac{n}{2}+2 k\right)$, then for $k \leq s \leq \frac{n}{2}+k+1$ (resp. $\left.2 k \leq s \leq \frac{n}{2}+2 k+1\right)$, we have the following decomposition:

$$
H^{s-k}(\eta)=\operatorname{Im} D_{s} \oplus \operatorname{Ker} D^{*} .
$$

The range $\operatorname{Im} D_{s}$ is closed and the sum is orthogonal w.r.t. the $L^{2}$ scalar product, furthermore, the range is closed in $H^{s-k}(\eta)$.

As in the case of self adjoint elliptic operators with smooth coefficients, from self adjoint elliptic operators, with coefficients in $H^{m}$, one can build Fredholm operators.

Corollary A.2.3. Let $D$ be a self adjoint operator, $D \in \operatorname{Ell}^{m}(\xi, \xi)$ of order $k$, with $m>\frac{n}{2}+k$. For $k \leq s \leq \frac{n}{2}+k+1$, the operator $D_{s}: H^{s}(\xi) \rightarrow H^{s-k}(\eta)$ is a Fredholm operator.

Proof. From Theorem A.2.2, we know that $\operatorname{Im} D_{s}$ is closed. On the other hand, one can check that the kernel $\operatorname{Ker} D_{s}$ is finite dimensional (see e.g. [C], Theorem 3.8) and hence $D_{s}$ is a Fredholm operator (see e.g. [G] Chap. I, Par. 4 for a definition of a Fredholm operator).

Self adjoint elliptic operators with $H^{m}$ coefficients and $m$ large enough have the same spectral properties as the ones with smooth coefficients as shown in this theorem.

Theorem A.2.4. A self adjoint operator $D \in \operatorname{Ell}^{m}(\xi, \xi)$ of order $k$ with $m>\frac{n}{2}+k$ has purely discrete spectrum $\left\{\lambda_{n}, n \in \mathbb{N}\right\}$ and that there exists a constant $C>0$ and an exponent $\delta>0$ with $\left|\lambda_{n}\right| \geq C n^{\delta}$ for large enough $n$.

Proof. We can apply exactly the same proof as in Lemma 1.6.3 [G] since it is based on the previous results of this appendix.

Since the spectral properties for self adjoint elliptic operators with $H^{m}$ coefficients are the same as that of self adjoint elliptic operators with smooth coefficients, we can apply the procedure described in [AJPS] to define regularised determinants of self adjoint elliptic operators with coefficients in $H^{m}$ ( $m$ chosen large enough), so that following [AJPS] Chap. I.5, for a positive self adjoint operator $A \in \mathrm{Ell}^{m}(\xi)$, we can define $\operatorname{det} A$ and for an operator $B \in \operatorname{Inj}^{m}(\xi, \eta)$, we can $\operatorname{define} \operatorname{det} B=\left(\operatorname{det} B^{*} B\right)^{1 / 2}$.

\section{Appendix A.3: I.L.H. Structures}

The following definitions can be found in [B, FT, O] for example.

Recall that a topological linear space $E$ is an I.L.H. space if $E=\bigcap_{i \in \mathbb{N}} H_{i}$, where $H_{\imath}, i \in \mathbb{N}$ are Hilbert spaces such that $H_{\imath} \subset H_{\jmath}$ for $j \leq i$, and the inclusion is a bounded linear operator.

Definition A.3.1. A topological space $X$ is an I.L.H. $C^{k}$ manifold modelled on an I.L.H. space $F$ if it satisfies conditions 1$), 2$ ), 3):

1) $X$ is a projective limit of Hilbert manifolds $X_{i}$ modelled on $H_{i}$ such that $X_{h} \subset X_{\imath}$ for $i \leq j$ and such that for all $x \in X$, there is a neighborhood $U_{i}(x) \in X_{\imath}, a$ 
neighborhood $V_{i}$ of 0 in $H_{\imath}$ and a map $\psi_{\imath}: U_{i}(x) \subset X_{\imath} \rightarrow V_{\imath} \subset H_{\imath}$ such that $U_{\imath+1}(x) \subset U_{\imath}(x)$ and $\psi_{i} / U_{\imath+1}(x)=\psi_{\imath+1}$.

2) $\psi_{\imath} \circ \psi_{\jmath}^{-1}: V_{j} \rightarrow U_{\imath}$ is $C^{k}$ between Hilbert spaces.

3) The inductive limit of $U_{i}(x), i \in \mathbb{N}$ is a neighborhood of $x$ in $X$.

If it is $C^{k}$ for all $k$, then it is a $C^{\infty}$ I.L.H. manifold.

Remark. We have included condition 3) in the definition of an I.L.H. manifold which makes it a strong I.L.H. manifold, according to the usual convention, so that all I.L.H. manifolds considered here are in fact strong I.L.H. manifolds.

A typical example of such a $C^{\infty}$ I.L.H. manifold is the manifold

$$
\mathscr{L b}(M)=\left\{g \in C^{\infty}\left(S^{2} T^{*}\right), \operatorname{det} g>0\right\}=\bigcap_{\imath \in \mathbb{N}} \mathscr{L}^{\imath}(M),
$$

where $M$ is as before a $C^{\infty}$ riemannian boundaryless manifold. In order to define the notion of I.L.H. Lie group, we need that of I.L.H. map.

Definition A.3.2. a) A map $\phi: X \rightarrow Y$ between I.L.H. manifolds of class $C^{k}$ is an I.L.H. map of class $C^{k}$ if it is an inductive limit of $C^{k}$ maps $\phi_{\imath}: X_{\jmath(i)} \rightarrow Y_{i}$ for a certain $j(i)$ such that $\phi_{i} / X_{j(\imath+1)}=\phi_{i+1}$. It is $C^{\infty}$ if it is $C^{k}$ for all $k$. It is of order smaller or equal to $k$ if $j(i)$ can be chosen smaller or equal to $i+k$ for all $i$.

b) An I.L.H. Lie group is a $C^{\infty}$ I.L.H. manifolds such that all operations are $C^{\infty}$ I.L.H. maps.

c) Let $X$ be a $C^{\infty}$ I.L.H. manifold, $G$ an I.L.H. Lie group acting on $X$ and $X / G$ an I.L.H. manifold. Let $\pi: X \rightarrow X / G$ denote the canonical projection. The triple $(\pi, X, X / G)$ defines an I.L.H. fibre bundle if and only if the transition functions are $C^{\infty}$ I.L.H.

A typical example of an I.L.H. Lie group is (with the same notations as above):

$$
\begin{aligned}
\mathscr{D}(M) & \equiv\left\{f \in C^{\infty}(M \times M), \text { such that } f \text { has an inverse and } f^{-1} \in C^{\infty}(M \times M)\right\} \\
& =\bigcap_{s \in \mathbb{N}} \mathscr{D}^{s}(M) .
\end{aligned}
$$

It is indeed a $C^{\infty}$ I.L.H. manifold modelled on $C^{\infty}(T M)$ (see e.g. [O]). Moreover for each $k$, there is an $s(k)>k$ such that the left multiplication defined for $h \in$ by:

$$
\begin{aligned}
\mathscr{D}^{s(k)}(M) & \rightarrow \mathscr{D}^{k}(M), \\
f & \rightarrow h \circ f,
\end{aligned}
$$

and such that the inversion:

$$
\begin{aligned}
\mathscr{D}^{s(k)}(M) & \rightarrow \mathscr{D}^{k}(M) \\
f & \rightarrow f^{-1}
\end{aligned}
$$

are of class $C^{k}$. Furthermore, the right multiplication defined for $h \in \mathscr{D}^{k}(M)$ by:

$$
\begin{aligned}
\mathscr{D}^{k}(M) & \rightarrow \mathscr{Q}^{k}(M) \\
f & \rightarrow f \circ h
\end{aligned}
$$

is $C^{\infty}$.

Another similar example is the group given by the semi-direct product $\mathscr{Q}(M) \odot$ $\mathscr{W}^{\prime}(M)$, where $\mathscr{W}(M)=\left\{e^{\phi}, \phi \in C^{\infty}(M, \mathbb{R})\right\}=\bigcap_{s \in \mathbb{N}} \mathscr{W}^{s}(M)$. 
Acknowledgements. I would like to thank D. Bennequin, T. Delzant and M. Slupinski for very enlightening discussions and S. Scarlatti for his constructive remarks concerning this paper. Special thanks to Z. Jaskolski who helped me a great deal making very relevant criticisms concerning certain points of the paper and pointing out to me some very useful references. I am also grateful to the referees of this paper for making very helpful comments.

\section{References}

[AGN] Alvarez Gaumé, L., Nelson, P.: Riemann surfaces and string theory. In Supergeometry, Supergravity and Superstrings '86, Singapore: World Scientific, 1986

[AHKPS] Albeverio, S., Hoegh-Krohn, R., Paycha, S., Scarlatti, S.: A global and stochastic approach to bosonic strings and associated quantum fields. Acta Applicandae Mathematicae, Vol. 26, No. 2, 103-195 (1992)

[AJPS] Albeverio, S., Jost, J., Paycha, S.,Scarlatti, S.: A mathematical introduction to string theory - variational problems, geometric and probabilistic methods. To appear

[B] Bourbaki, N.: Variétés différentielles et analytiques. Fascicule de résultats par. 1 à 7

[Bo] Bourguignon, J.P.: L'espace des structure Riemanniennes d'une variété compacte. Thèse Paris VII, (1974)

[Br] Bredon, G.E.: Introduction to compact transformation groups. N.Y.: Academic Press, 1972

[BeEb] Berger, M., Ebin, D.: Some decompositions of the space of symmetric tensors on a Riemannian manifold. J. Diff. Geom. n. 3, 379-392 (1969)

[BV] Babelon, O., Viallet, C.M.: The gometrical interpretation of the Faddeev-Popov determinant. Phys. Lett. 85B, 246 (1979)

[C] Cantor, M.: Elliptic operators and the decomposition of tensor fields. Bull. Am. Soc. Vol. 5, n. 3, 235-262 (1981)

[dHP] d'Hooker, E., Phong, D.: Multiloop amplitudes for the bosonic Polyakov string. Nucl. Phys. B 269, 205 (1986)

[E] Ebin, D.: The manifold of Riemannian metrics. Proc. Symp. Pure Math., A.M.S. 15, 11-40, (1970)

[F] Freed, D.S.: Determinants. Torsion and Strings. Commun. Math. Phys. 107, 483-513 (1986)

[FM] Fischer, A.E., Marsden, J.: The manifold of conformally equivalent metrics. Can. J. Math. 29, 193-209 (1977)

[FP] Faddeev, L.D., Popov, V.N.: Feynman diagrams for the Yang-Mills fields. Phys. Lett. 25B, 30 (1967)

[FT] Fischer, A.E., Tromba, A.J.: On a purely Riemannian proof of the structure and dimension of the unramified moduli space of a compact Riemann surface. Math. Annalen 267, 311345 (1984)

[FU] Freed, D.S., Uhlenbeck, K.K.: Instantons and Four manifolds. Berlin, Heidleberg, New York: Springer 1984

[H] Hamilton, R.S.: The inverse function theorem of Nash-Moser. Bull. Amer. Math. Soc. 7, N.1, 65-221 (1982)

[IM] Isenberg, J., Marsden, J.: A slice theorem for the space of solutions of Einstein's equations. Phys. Rep. 89, 179-222 (1982)

[J] Jaskolski, Z.: The geometric interpretation of the Faddeev-Popov determinant in Polyakov's theory of random surfaces. J. Math. Phys. 27, 2570 (1986);

The integration of $G$-invariant functions and the Faddeev-Popov procdure. Commun. Math. Phys. 111, 439-468 (1987)

[K] Killingback, T.P.: Global aspects of fixing the gauge in the Polyakov string and Einstein gravity. Commun. Math. Phys. 100, 267-277 (1985)

[KR] Kondracki, W., Rogulski, J.: On the stratification of the orbit space. Preprint n. IMPAN 2/84/182 of the Institute of Math. Polish Academy of Sciences

[MN] Moore, G., Nelson, P.: Measure for moduli. Nucl. Phys. B 266, 58 (1986)

[MV] Mitter, P.K., Viallet, C.M.: On the bundle of connections and the gauge orbit manifold in Yang-Mills theory, Commun. Math. Phys. 79, 457-472 (1981)

[NR] Narashiman, M.S., Ramadas, T.R.: Geometry of $S U(2)$ gauge fields. Commun. Math. Phys. 67, 121-136 (1979) 
[O] Omori, H.: On the group of diffeomorphisms on a compact manifold. Proc. Symp. Pure Math. A.M.S. 15, 167-183 (1970)

[Pa] Paycha, S.: The Faddeev-Popov procedure and application to bosonic strings: An infinite dimensional point of view. Commun. Math. Phys. 147, 163-180 (1992)

[P] Polchinski, J.: From Polyakov to moduli. In: Mathematical aspects of string theory. Proceedings of San Diego Conference, July 1986, Singapore: World Scientific, 1987, pp. 13-28

[S] Singer, I.M.: Some remarks on the Gribov ambiguity. Commun. Math. Phys. 60 (1978)

[V] Varadarajan, V.S.: Lie groups, Lie algebras and their representations. New York: PrenticeHall 1974

Communicated by H. Araki 
